\title{
The Siegel-Walfisz theorem for Rankin-Selberg $L$-functions associated with two cusp forms
}

by

\author{
YUMiko ICHIHARA (Nagoya)
}

1. Introduction. For the coefficients of a cusp form of integral weight $k$ for $\mathrm{SL}_{2}(\mathbb{Z})$, Perelli [13] showed an analogue of the Siegel-Walfisz prime number theorem. His result was proved by using Rankin-Selberg $L$-functions $L_{f \otimes f}(s, \chi)$ (see the definition below). The aim of this paper is to prove an analogue of the Siegel-Walfisz theorem for the product of the Fourier coefficients of two cusp forms. It is obtained through the investigation of Rankin-Selberg $L$-functions associated with two cusp forms for $\mathrm{SL}_{2}(\mathbb{Z})$.

Let $S_{k}(\Gamma)$ be the space of cusp forms of weight $k$ for $\Gamma=\mathrm{SL}_{2}(\mathbb{Z})$. Let $f \in S_{k}(\Gamma)$ and $g \in S_{l}(\Gamma)$ be normalized Hecke eigenforms. We write their Fourier series expansions at the cusp as

$$
f(z)=\sum_{n=1}^{\infty} a_{n} e^{2 \pi i n z}, \quad g(z)=\sum_{n=1}^{\infty} b_{n} e^{2 \pi i n z} .
$$

The Rankin-Selberg L-function associated with $f$ and $g$ is defined as

$$
\begin{aligned}
L_{f \otimes g}(s)= & \prod_{p}\left(1-\alpha_{p} \beta_{p} p^{-s}\right)^{-1}\left(1-\alpha_{p} \bar{\beta}_{p} p^{-s}\right)^{-1} \\
& \times\left(1-\bar{\alpha}_{p} \beta_{p} p^{-s}\right)^{-1}\left(1-\overline{\alpha_{p} \beta_{p}} p^{-s}\right)^{-1},
\end{aligned}
$$

where $p$ runs through all prime numbers, $\alpha_{p}$ and $\beta_{p}$ appear in the Euler products of $L_{f}(s)$ and $L_{g}(s)$ as follows:

$$
\begin{aligned}
& L_{f}(s)=\sum_{n=1}^{\infty} \frac{a_{n}}{n^{s}}=\prod_{p}\left(1-\alpha_{p} p^{-s}\right)^{-1}\left(1-\bar{\alpha}_{p} p^{-s}\right)^{-1}, \\
& L_{g}(s)=\sum_{n=1}^{\infty} \frac{b_{n}}{n^{s}}=\prod_{p}\left(1-\beta_{p} p^{-s}\right)^{-1}\left(1-\bar{\beta}_{p} p^{-s}\right)^{-1},
\end{aligned}
$$

in other words $\alpha_{p}$ and $\beta_{p}$ are complex numbers satisfying $\alpha_{p}+\bar{\alpha}_{p}=a_{p}$,

2000 Mathematics Subject Classification: 11F30, 11F66. 
$\left|\alpha_{p}\right|=p^{(k-1) / 2}$ and $\beta_{p}+\bar{\beta}_{p}=b_{p},\left|\beta_{p}\right|=p^{(l-1) / 2}$. (Here, $\bar{z}$ means the complex conjugate of $z$.) We also define the twisted Rankin-Selberg L-function as

$$
\begin{aligned}
L_{f \otimes g}(s, \chi)= & \prod_{p}\left(1-\alpha_{p} \beta_{p} \chi(p) p^{-s}\right)^{-1}\left(1-\alpha_{p} \bar{\beta}_{p} \chi(p) p^{-s}\right)^{-1} \\
& \times\left(1-\bar{\alpha}_{p} \beta_{p} \chi(p) p^{-s}\right)^{-1}\left(1-\overline{\alpha_{p} \beta_{p}} \chi(p) p^{-s}\right)^{-1},
\end{aligned}
$$

where $\chi$ is a Dirichlet character modulo $d \geq 1$. For $\operatorname{Re}(s)>(k+l) / 2$, it is clear that

$$
L_{f \otimes g}(s, \chi)=L\left(2 s-k-l+2, \chi^{2}\right) L_{f, g}(s, \chi),
$$

where

$$
L_{f, g}(s, \chi)=\sum_{n=1}^{\infty} a_{n} b_{n} \chi(n) n^{-s},
$$

and $L(s, \chi)$ is the Dirichlet $L$-function attached to $\chi$.

In Section 2, we consider the zero-free region of $L_{f \otimes g}(s, \chi)$. When $\chi$ is a non-primitive character modulo $d$ and $\chi^{*}$ is the primitive character which induces $\chi$, then $L_{f \otimes g}(s, \chi)$ can be expressed as follows:

$$
\begin{aligned}
L_{f \otimes g}(s, \chi)= & L_{f \otimes g}\left(s, \chi^{*}\right) \prod_{p \mid d}\left(1-\alpha_{p} \beta_{p} \chi(p) p^{-s}\right)\left(1-\alpha_{p} \bar{\beta}_{p} \chi(p) p^{-s}\right) \\
& \times\left(1-\bar{\alpha}_{p} \beta_{p} \chi(p) p^{-s}\right)\left(1-\overline{\alpha_{p} \beta_{p}} \chi(p) p^{-s}\right) .
\end{aligned}
$$

Therefore, it is enough to consider the zero-free region of Rankin-Selberg $L$-functions with primitive characters. In the classical argument for the zerofree regions of Dirichlet $L$-functions, and also in Perelli's proof for the case $f=g$ of Rankin-Selberg $L$-functions, positivity of some quantities plays an essential role. Such positivity, however, is not valid for $f \neq g$. In this paper we develop a new approach which is a variant of the classical argument but without using the positivity, and we will obtain the following theorem. Hereafter we write $s=\sigma+i t$.

Theorem 1. Let $f \in S_{k}(\Gamma)$ and $g \in S_{l}(\Gamma)$ be normalized Hecke eigenforms. There exists a positive constant $c=c(f, g)$ such that $L_{f \otimes g}(s, \chi) \neq 0$ in the region

$$
\sigma>\frac{k+l}{2}-\frac{c}{\log (d(|t|+2))},
$$

where $\chi$ is a primitive Dirichlet character modulo $d \geq 1$. However, there is at most one exceptional zero $(<(k+l) / 2)$ which is real and simple in the case where $\chi$ is a real non-principal character.

Consider the set $S$ of functions $L_{f \otimes g}(s, \chi)$ where $\chi$ runs through all real primitive characters modulo $d \geq 2$. Then there exists a positive constant $c$ such that in $S$ there is at most one $L_{f \otimes g}(s, \chi)$ which has a zero in the above region, and this zero is unique, real and simple. 
The exceptional zero in Theorem 1 is called the Siegel zero. Concerning the Siegel zero for the $L$-functions for GL(2) or GL(3), there is a work of Hoffstein-Lockhart [7], with appendix by D. Goldfeld, J. Hoffstein and D. Lieman. This article shows that, in many cases, $L_{f \otimes f}(s, \chi)$ attached to a Maass form $f$ does not have the Siegel zero. For certain $L$-series for GL(3), Hoffstein-Ramakrishnan [8] show the non-existence of the Siegel zero in several cases. We cannot apply their result to $L_{f \otimes g}(s, \chi), f \neq g$, because it is an $L$-series for GL(4). Though $L_{f \otimes f}(s, \chi)$ is an $L$-series on GL(3), this case needs Hypothesis 6.1 in Hoffstein-Ramakrishnan [8] for the proof of the non-existence of the Siegel zero. Hence, investigating the Siegel zero for $L_{f \otimes g}(s, \chi)$ is still of interest. Theorem 2 is Siegel's theorem for $L_{f \otimes g}(s, \chi)$. Siegel's theorem for $L_{f \otimes f}(s, \chi)$ in Perelli's paper was proved by using a result of Perelli-Puglisi [14]. But the proof in [14] is incorrect, as mentioned in Carletti-Monti Bragadin-Perelli [1]. This mistake was also mentioned by Golubeva-Fomenko [5], who suggested an alternative way of proving Siegel's theorem for $L_{f \otimes f}(s, \chi)$, but their treatment seems to be a little rough.

The next theorem gives a zero-free region of Siegel's type on the real axis for $L_{f \otimes g}(s, \chi)$. The basic idea of the proof is similar to Golubeva-Fomenko [5]. We could get a sharp estimate of the twisted coefficient sum, which is better than that used in [5], but we do not do it in this paper, because we are able to obtain Siegel's theorem for $L_{f \otimes g}(s, \chi)$ without this sharp estimate. Our proof includes the case of $f=g$, hence covers Perelli's assertion.

Theorem 2 (An analogue of Siegel's theorem). Let $f$ and $g$ be as in Theorem 1 and $\chi$ a real primitive character modulo $d \geq 2$. Then, for any $\varepsilon>0$, there exists a positive constant $c(\varepsilon)=c(\varepsilon, f, g)$ such that $L_{f \otimes g}(s, \chi) \neq$ 0 in the region

$$
\sigma>\frac{k+l}{2}-\frac{c(\varepsilon)}{d^{\varepsilon}} .
$$

Professor A. Perelli informed us that now he has an idea, similar to the method of proof in this paper, of proving an analogue of Siegel's theorem for $L_{f \otimes f}(s, \chi)$.

In Section 3, taking account of Theorems 1 and 2, we will prove the following Theorem 3, which is an analogue of the Siegel-Walfisz prime number theorem

$$
\sum_{\substack{n \leq x \\ n \equiv a(\bmod d)}} \Lambda(n)=\frac{x}{k \varphi(d)}+O(x \exp (-c \sqrt{\log x})),
$$

where $\Lambda(n)$ is the von Mangoldt function. This is the main theorem in this paper.

Theorem 3. Let $f \in S_{k}(\Gamma)$ and $g \in S_{l}(\Gamma)$ be normalized Hecke eigenforms and assume $f \neq g$. Denote by $a_{n}$ and $b_{n}$ the nth Fourier coefficients 
of $f$ and $g$ respectively. Let $a, d$ be positive integers with $(a, d)=1$, and $M$ be a positive number. Then there exists a positive constant $c=c(M)$ which depends on $M$ such that

$$
\sum_{\substack{p \leq x \\ p \equiv a(\bmod d)}} a_{p} b_{p}=O\left(x^{(k+l) / 2} \exp (-c \sqrt{\log x})\right)
$$

for $d \leq(\log x)^{M}$.

The difference as compared with Perelli's result [13] is that the main term does not appear in the case of $f \neq g$. Perelli's result implies that if $f=g$ then

$$
\sum_{\substack{n \leq x \\ n \equiv a(\bmod d)}} a_{n}^{2} \Lambda(n)=\frac{x^{k}}{k \varphi(d)}+O\left(x^{k} \exp (-c \sqrt{\log x})\right) .
$$

This difference is caused by the fact that $L_{f \otimes f}(s)$ has a simple pole at $s=k$, while $L_{f \otimes g}(s), f \neq g$, is an entire function.

Throughout this paper, $c$ denotes a positive constant, not necessarily the same at each occurrence.

The author would like to express her deep gratitude to Professor Yoshio Tanigawa for his very important advice indicating how to construct an argument without using the positivity, and his kindness. She is also grateful to Professor Kohji Matsumoto for valuable suggestions, discussions and encouragement, and to Professor A. Perelli for useful information and advice. She expresses her thanks to Professor A. Ivić and Professor Hirotada Naito for advice and comments, to Professor Tohru Uzawa and Professor Fumihiro Sato for discussions and valuable opinions, and also to the referee for valuable comments.

2. On the zeros of Rankin-Selberg $L$-functions. First, we recall several known results on $L_{f \otimes g}(s, \chi)$ which will be used later.

The Rankin-Selberg $L$-function is defined in (1.3). The functional equation of $L_{f \otimes g}(s, \chi)$ was given by Li [9]. He considered the Rankin-Selberg $L$-function attached to two cusp forms $F_{1}(\tau)$ and $F_{2}(\tau)$. Here we apply Li's result to

$$
F_{1}(\tau)=\sum_{n=1}^{\infty} a_{n} e^{2 \pi i n \tau}, \quad F_{2}(\tau)=\sum_{n=1}^{\infty} \overline{b_{n} \chi(n)} e^{2 \pi i n \tau} .
$$

Then $F_{1}$ is an element of $S_{k}(\Gamma)$, and $F_{2}$ is a newform of type $\left(l, \chi^{2}, d^{2}\right)$. Li's work shows (see Theorem 2.2 of $\mathrm{Li}[9]$ ) that the following functional 
equation holds for Rankin-Selberg $L$-functions. If we put

then we have

$$
\begin{aligned}
\Phi_{f \otimes g}(s, \chi)= & \left(\frac{2 \pi}{d}\right)^{-2 s} \Gamma\left(s+\frac{k-l}{2}\right) \\
& \times \Gamma\left(s+\frac{k+l}{2}-1\right) L_{f \otimes g}\left(s+\frac{k+l}{2}-1, \chi\right),
\end{aligned}
$$

$$
\Phi_{f \otimes g}(s, \chi)=C_{\chi} \Phi_{f \otimes g}(1-s, \bar{\chi})
$$

where $k \geq l, C_{\chi}$ is a constant which depends on $\chi$ and $\left|C_{\chi}\right|=1$. Without loss of generality, we may assume that $k \geq l$. Other important results are that the function $L_{f \otimes g}(s+(k+l) / 2-1)$ is entire when $f \neq g$, while it has a simple pole at $s=1$ when $f=g$. Also, the function $L_{f \otimes g}(s+(k+l) / 2-1, \chi)$ is entire for any $f$ and $g$ when $\chi$ is a primitive character modulo $d \geq 2$.

The following facts can be easily verified by using the functional equation (2.1) and the definition (1.3), where $\chi$ is a primitive character modulo $d \geq 1$.

1. $L_{f \otimes g}(s, \chi) \neq 0$ for $\operatorname{Re}(s)>(k+l) / 2$.

2. $L_{f \otimes g}(s, \chi)$ has a zero of order two at $s=-n(n \in \mathbb{N} \cup\{0\})$.

3. $L_{f \otimes g}(s, \chi)$ has a simple zero at $s=l-n-1(n \in \mathbb{N} \cup\{0\}, 0 \leq n \leq l-2)$.

The zeros of $L_{f \otimes g}(s, \chi)$ in $\operatorname{Re}(s)<(k+l) / 2-1$ are called the trivial zeros, and those in $(k+l) / 2-1 \leq \operatorname{Re}(s) \leq(k+l) / 2$ are called the non-trivial zeros.

Next, Perelli's general result [12] is also applicable to the case of RankinSelberg $L$-functions. Manin-Pančiškin [10] studied the functional equations for the twisted Rankin-Selberg $L$-functions when the moduli of Dirichlet characters are prime powers. However, the argument in 2.2 and 2.3 of their paper is valid for any modulus $d \geq 1$. Hence, 2.3(7) of their paper and the integral expression of Epstein-Siegel zeta functions (see Siegel [15], p. 53) show that Rankin-Selberg $L$-functions satisfy (A3) of Perelli [12]. Therefore, all conditions of Perelli [12] are satisfied by Rankin-Selberg $L$-functions. Theorem 1 of Perelli [12] and its corollary state the following facts. Let

$N_{L}(T)=\sharp\left\{\varrho \in \mathbb{C}\left|L_{f \otimes g}\left(\varrho+\frac{k+l}{2}-1, \chi\right)=0, \operatorname{Re}(\varrho) \geq 0,\right| \operatorname{Im}(\varrho) \mid \leq T\right\}$.

Then

$$
N_{L}(T)=\frac{2}{\pi}\left\{2 T \log T-2 T+T \log \frac{d^{2}}{4 \pi^{2}}\right\}+O(\log d T),
$$

where $T \geq 2$. It is also known by Perelli [12] that

$$
N_{L}(T+H)-N_{L}(T) \ll(H+1) \log d T,
$$

where $H \ll T$. 
Proof of Theorem 1. We prove the theorem in the case of $f \neq g$ because the proof for $f=g$ was already completed in Perelli [13]. We first assume $d \geq 2$. Let

$$
\Psi_{f \otimes g}(\sigma+i t, \chi)=-3 \frac{L_{f \otimes g}^{\prime}}{L_{f \otimes g}}\left(\sigma, \chi_{0}\right)-4 \frac{L_{f \otimes g}^{\prime}}{L_{f \otimes g}}(\sigma+i t, \chi)-\frac{L_{f \otimes g}^{\prime}}{L_{f \otimes g}}\left(\sigma+2 i t, \chi^{2}\right)
$$

for $\sigma>(k+l) / 2$, where $\chi_{0}$ is the principal character modulo $d$. Considering this type of auxiliary function is an orthodox method of studying zero-free regions of $L$-functions. In fact, functions of this type were considered in the study of zero-free regions for Dirichlet $L$-functions and also for $L_{f \otimes f}(s, \chi)$. In those cases, the real parts of the corresponding auxiliary functions are non-negative, which is the key fact to the proof. However, in the present case, since

$$
\begin{aligned}
& \operatorname{Re}\left(\Psi_{f \otimes g}(\sigma+i t, \chi)\right) \\
& =\sum_{(p, d)=1} \sum_{m=1}^{\infty}\left(\alpha_{p}^{m}+\bar{\alpha}_{p}^{m}\right)\left(\beta_{p}^{m}+\bar{\beta}_{p}^{m}\right) p^{-m \sigma} \log p \cdot\left(3+4 \cos \theta_{m, p}+\cos 2 \theta_{m, p}\right)
\end{aligned}
$$

where $\cos \theta_{m, p}=\operatorname{Re}\left(\chi\left(p^{m}\right) p^{-m i t}\right)$, it seems impossible to prove that $\operatorname{Re}\left(\Psi_{f \otimes g}(\sigma+i t, \chi)\right) \geq 0$. For this reason, we cannot use the classical positivity argument in our case. The following method, indicated by Professor Y. Tanigawa, is effective for our purpose. Using the relation between the arithmetic and geometric means we have

$$
\begin{aligned}
\left(\alpha_{p}^{m}+\bar{\alpha}_{p}^{m}\right) & \left(\beta_{p}^{m}+\bar{\beta}_{p}^{m}\right) p^{-m \sigma} \\
& \geq-\frac{1}{2}\left\{\left(\alpha_{p}^{m}+\bar{\alpha}_{p}^{m}\right)^{2} p^{-m(\sigma+(k-l) / 2)}+\left(\beta_{p}^{m}+\bar{\beta}_{p}^{m}\right)^{2} p^{-m(\sigma-(k-l) / 2)}\right\}
\end{aligned}
$$

hence

$$
\geq-\frac{1}{2}\left\{\operatorname{Re}\left(\Psi_{f \otimes f}\left(\sigma+\frac{k-l}{2}+i t, \chi\right)\right)+\operatorname{Re}\left(\Psi_{g \otimes g}\left(\sigma+\frac{l-k}{2}+i t, \chi\right)\right)\right\} .
$$
Then

$$
-\operatorname{Re}\left(\Psi_{f \otimes f}\left(\sigma+\frac{k-l}{2}+i t, \chi\right)\right)>\frac{-3}{\sigma-(k+l) / 2}+O(\log (d(|t|+2)))
$$

in $(k+l) / 2<\sigma \leq(k+l) / 2+1$. This inequality can be derived by the standard argument described in Perelli [13] and Section 14 of Davenport [2] (cf. Lemma in the proof of Theorem 2 in Perelli [12]). The same type of inequality can be obtained for $\operatorname{Re}\left(\Psi_{g \otimes g}(\sigma-(l-k) / 2+i t, \chi)\right)$. Using (2.4), we can show that, in $(k+l) / 2<\sigma \leq(k+l) / 2+1$,

$$
\operatorname{Re}\left(\Psi_{f \otimes g}(\sigma+i t, \chi)\right)>\frac{-3}{\sigma-(k+l) / 2}+O(\log (d(|t|+2))) .
$$


Next, we show an upper bound of $\operatorname{Re}\left(\Psi_{f \otimes g}(\sigma+i t, \chi)\right)$. We recall that if $\chi$ is a primitive character, then $L_{f \otimes g}(s+(k+l) / 2-1, \chi)$ is an entire function. By the Lemma in the proof of Theorem 2 in Perelli [12] and the method of Section 14 of Davenport [2], we get the following facts in $(k+l) / 2<\sigma \leq$ $(k+l) / 2+1$ :

$$
\begin{gathered}
\operatorname{Re}\left(-\frac{L_{f \otimes g}^{\prime}}{L_{f \otimes g}}\left(\sigma, \chi_{0}\right)\right) \leq c \log d \\
\operatorname{Re}\left(-\frac{L_{f \otimes g}^{\prime}}{L_{f \otimes g}}(\sigma+i t, \chi)\right) \leq-\sum_{|\gamma-t|<1} \frac{1}{\sigma-\beta}+O(\log (d(|t|+2))),
\end{gathered}
$$

and

$$
\operatorname{Re}\left(-\frac{L_{f \otimes g}^{\prime}}{L_{f \otimes g}}\left(\sigma+2 i t, \chi^{2}\right)\right) \leq c \log (d(|t|+1)),
$$

where $\varrho=\beta+i \gamma$ runs through non-trivial zeros of $L_{f \otimes g}(\sigma+i t, \chi)$. We fix one such zero. We replace the terms $-(\sigma-\beta)^{-1}$ on the right-hand side of (2.8) by 0 , for all zeros except this fixed $\varrho$. Then from (2.7) and (2.9), we get

$$
\operatorname{Re}\left(\Psi_{f \otimes g}(\sigma+i \gamma, \chi)\right)<-\frac{4}{\sigma-\beta}+O(\log (d(|\gamma|+2)))
$$

for $(k+l) / 2<\sigma \leq(k+l) / 2+1$. Therefore, using (2.6), we get

$$
-\frac{4}{\sigma-\beta}+O(\log (d(|\gamma|+2)))>-\frac{3}{\sigma-(k+l) / 2}
$$

for $(k+l) / 2<\sigma \leq(k+l) / 2+1$. Because this inequality is the same as in Davenport [2], the rest of the proof in this case proceeds along the same lines as in [2].

Second step. We consider the case when $\chi$ is a primitive real character. Using (2.4), we get

$$
\begin{aligned}
-\operatorname{Re} & \left(\Psi_{f \otimes f}\left(\sigma+\frac{k-l}{2}+i t, \chi\right)\right) \\
& >\frac{-3}{\sigma-(k+l) / 2}-\operatorname{Re}\left(\frac{1}{\sigma+2 i t-(k+l) / 2}\right)+O(\log (d(|t|+2)))
\end{aligned}
$$

in $(k+l) / 2<\sigma \leq(k+l) / 2+1$. This inequality is obtained by the argument which shows (2.5). Similarly to $(2.6)$ we get, in $(k+l) / 2<\sigma \leq(k+l) / 2+1$,

$$
\begin{aligned}
& \operatorname{Re}\left(\Psi_{f \otimes g}(\sigma+i t, \chi)\right) \\
& >\frac{-3}{\sigma-(k+l) / 2}-\operatorname{Re}\left(\frac{1}{\sigma+2 i t-(k+l) / 2}\right)+O(\log (d(|t|+2))) .
\end{aligned}
$$


Because (2.7)-(2.10) are also true in this case, we get, using (2.12),

$$
-\frac{4}{\sigma-\beta}+O(\log (d(|\gamma|+2)))>-\frac{3}{\sigma-(k+l) / 2}-\operatorname{Re}\left(\frac{1}{\sigma+2 i \gamma-(k+l) / 2}\right)
$$

for $(k+l) / 2<\sigma \leq(k+l) / 2+1$. We can get a zero-free region in the same way as in the first case, except for the region close to the real axis.

We now consider the zero-free region close to the real axis. We again use the relation between the arithmetic and geometric means to get

$$
\begin{aligned}
& \left|\left(\alpha_{p}^{m}+\bar{\alpha}_{p}^{m}\right)\left(\beta_{p}^{m}+\bar{\beta}_{p}^{m}\right) \chi^{m}(p) p^{-m \sigma}\right| \\
& \quad \leq \frac{1}{2}\left\{\left(\alpha_{p}^{m}+\bar{\alpha}_{p}^{m}\right)^{2} p^{-m(\sigma+(k-l) / 2)}+\left(\beta_{p}^{m}+\bar{\beta}_{p}^{m}\right)^{2} p^{-m(\sigma+(l-k) / 2)}\right\} .
\end{aligned}
$$

Therefore

$$
\begin{aligned}
-\frac{L_{f \otimes g}^{\prime}}{L_{f \otimes g}}(\sigma, \chi) & =\sum_{(p, d)=1} \sum_{m=1}^{\infty}\left(\alpha_{p}^{m}+\bar{\alpha}_{p}^{m}\right)\left(\beta_{p}^{m}+\bar{\beta}_{p}^{m}\right) \chi^{m}(p) p^{-m \sigma} \log p \\
& \geq \frac{1}{2}\left\{\frac{L_{f \otimes f}^{\prime}}{L_{f \otimes f}}\left(\sigma+\frac{k-l}{2}\right)+\frac{L_{g \otimes g}^{\prime}}{L_{g \otimes g}}\left(\sigma+\frac{l-k}{2}\right)\right\},
\end{aligned}
$$

where $\sigma>(k+l) / 2$. We recall that $L_{f \otimes f}(s)$ has a simple pole at $s=k$. Then

$$
\frac{L_{f \otimes f}^{\prime}}{L_{f \otimes f}}\left(\sigma+\frac{k-l}{2}\right)>\frac{-1}{\sigma-(k+l) / 2}+O(1) \quad((k+l) / 2<\sigma \leq(k+l) / 2+1) .
$$

Using this inequality and (2.8), we obtain the desired zero-free region for $L_{f \otimes g}(s, \chi)$ near the real axis, by the argument described in Section 14 of Davenport [2].

Third step. We consider the real zero in the case when $\chi$ is a real character modulo $d \geq 2$. We prove that the stated zero-free region includes at most one real zero of $L_{f \otimes g}(s, \chi)$. This can be proved easily in the same way as in Davenport [2]. We can show that the exceptional zero is unique for all characters modulo $d$ using the function $F(s)$ which appears in the proof of Theorem 2, and the argument is again similar to Davenport [2].

Fourth step. We consider the case $d=1$, that is, the zero free-region for $L_{f \otimes g}(s)$. In this case, we use the inequality (2.14) below, which corresponds to (2.11) in the case of $d \geq 2$. However we stress that (2.14) is not identical to the one in Davenport [2], hence we need an alternative argument.

In a way similar to $(2.12)$ we get

$$
\begin{aligned}
> & -\frac{3}{\sigma-(k+l) / 2}-\operatorname{Re}\left(\frac{4}{\sigma+i t-(k+l) / 2}\right)-\operatorname{Re}\left(\frac{1}{\sigma+2 i t-(k+l) / 2}\right) \\
& +O(\log (|t|+2))
\end{aligned}
$$


in $(k+l) / 2<\sigma<(k+l) / 2+1$, where

$$
\Psi_{f \otimes g}(\sigma+i t)=-3 \frac{L_{f \otimes g}^{\prime}}{L_{f \otimes g}}(\sigma)-4 \frac{L_{f \otimes g}^{\prime}}{L_{f \otimes g}}(\sigma+i t)-\frac{L_{f \otimes g}^{\prime}}{L_{f \otimes g}}(\sigma+2 i t) .
$$

Note that the existence of the poles at $s=k$ of $L_{f \otimes f}(s)$ and $s=l$ of $L_{g \otimes g}(s)$ yields the second term on the right-hand side of (2.13). Using the Lemma in the proof of Theorem 2 of Perelli [12], we get

$$
\begin{aligned}
& (2.14) \quad-\frac{4}{\sigma-\beta}+O(\log (|\gamma|+2)) \\
& >-\frac{3}{\sigma-(k+l) / 2}-\operatorname{Re}\left(\frac{4}{\sigma+i \gamma-(k+l) / 2}\right)-\operatorname{Re}\left(\frac{1}{\sigma+2 i \gamma-(k+l) / 2}\right) .
\end{aligned}
$$

For $\{s \in \mathbb{C} \mid \operatorname{Im}(s) \geq 1\}$, we replace the denominators of the second and third terms by 1 . Then we obtain the desired zero-free region in a way similar to that of Davenport [2].

We see that the required assertion is true for the non-trivial zeros which are near the real axis, say $|\gamma|<c_{0}$, since $L_{f \otimes g}((k+l) / 2) \neq 0$. We can prove the latter by applying the method of Ogg [11] to $F(s)=L_{f \otimes f}(s+k-1) \times$ $L_{f \otimes g}^{2}(s+(k+l) / 2-1) L_{g \otimes g}(s+l-1)$ (cf. the proof of Theorem 4 of [11]).

Lastly we have to consider the non-trivial zero $\varrho=\beta+i \gamma, c_{0} \leq|\gamma|<1$. Let $\sigma=(k+l) / 2+c_{1}, c_{1}=c_{0} / \lambda(\lambda>1)$. Using (2.14), we get

$$
-\frac{4}{\sigma-\beta}+c_{2}>-\frac{3}{c_{1}}-\frac{4 c_{1}}{c_{1}^{2}+c_{0}^{2}}-\frac{c_{1}}{c_{1}^{2}+4 c_{0}^{2}}>-\frac{1}{c_{1}}\left(3+\frac{4}{\lambda^{2}+1}+\frac{1}{4 \lambda^{2}+1}\right),
$$

where $\log (|\gamma|+2)<c_{2}$. We take $\lambda$ which is large enough to satisfy $3+$ $4 /\left(\lambda^{2}+1\right)+1 /\left(4 \lambda^{2}+1\right)<4$. Thus we obtain the zero-free region in this case.

Siegel's theorem gives the detailed information on the zero-free region of Dirichlet's $L$-functions on the real axis. We want to show its analogue for $L_{f \otimes g}(s, \chi)$. As mentioned in the introduction, Siegel's theorem for $L_{f \otimes f}(s, \chi)$ has not been proved completely. Therefore we do not exclude the case $f=g$ in the following proof. We need the estimates $L_{f \otimes g}(s, \chi) \ll d^{\varepsilon}$ and $L_{f \otimes g}^{\prime}(s, \chi) \ll d^{\varepsilon}$ for any $\varepsilon>0$. We can get them by Theorem 2 of Carletti-Monti Bragadin-Perelli [1] or by Phragmén-Lindelöf's theorem.

Golubeva-Fomenko [5] stated a certain estimate of $\sum_{n \leq x} a_{n}^{2} \chi(n)$, without detailed proof; they deduced from it the necessary estimates for $L_{f \otimes f}(s, \chi)$ and $L_{f \otimes f}^{\prime}(s, \chi)$. We can get an estimate of $\sum_{n \leq x} a_{n} b_{n} \chi(n)$, better than that of Golubeva-Fomenko [5] in the case of $f=g$, by using Hafner [6]. We will discuss this matter elsewhere, because such sharp estimates are not necessary for our present aim.

The base of the proof of Theorem 2 is Davenport [2]. There is a simple proof of Siegel's theorem for Dirichlet $L$-functions (see Goldfeld [4]), but 
we cannot apply that argument to $L_{f \otimes g}(s, \chi)$, because it has no pole at $s=(k+l) / 2$ in this case.

Proof of Theorem 2. Let $\chi_{i}(i=1,2)$ be primitive real characters modulo $d_{i} \geq 2$ such that $\chi_{1} \chi_{2}$ is a non-principal real character modulo $d_{1} d_{2}$. We consider the case of $f \neq g$. Let $F(s)$ be the meromorphic function defined by

$$
\begin{aligned}
F(s)= & L_{f \otimes f}(s+k-1) L_{f \otimes f}\left(s+k-1, \chi_{1}\right) L_{f \otimes f}\left(s+k-1, \chi_{2}\right) \\
& \times L_{f \otimes f}\left(s+k-1, \chi_{1} \chi_{2}\right) L_{f \otimes g}^{2}\left(s+\frac{k+l}{2}-1\right) \\
& \times L_{f \otimes g}^{2}\left(s+\frac{k+l}{2}-1, \chi_{1}\right) L_{f \otimes g}^{2}\left(s+\frac{k+l}{2}-1, \chi_{2}\right) \\
& \times L_{f \otimes g}^{2}\left(s+\frac{k+l}{2}-1, \chi_{1} \chi_{2}\right) L_{g \otimes g}(s+l-1) L_{g \otimes g}\left(s+l-1, \chi_{1}\right) \\
& \times L_{g \otimes g}\left(s+l-1, \chi_{2}\right) L_{g \otimes g}\left(s+l-1, \chi_{1} \chi_{2}\right),
\end{aligned}
$$

for $\sigma>1$. This definition is inspired by Ogg [11], who used the function $L_{f \otimes f}(s) L_{f \otimes g}^{2}(s) L_{g \otimes g}(s)$ in the proof that $L_{f \otimes g}(1) \neq 0$ (Thorem 4 of Ogg [11]). The function $F(s)$ has a pole of order two at $s=1$. Let $\lambda_{1}=\left.\left(F(s)(s-1)^{2}\right)^{\prime}\right|_{s=1}$ and $\lambda_{2}=\left.\left(F(s)(s-1)^{2}\right)\right|_{s=1}$. We investigate $F(s)-\lambda_{2}(s-1)^{-2}-\lambda_{1}(s-1)^{-1}$ as in Davenport [2]. In this procedure we use the estimates of $L_{f \otimes g}(s, \chi)$ and $L_{f \otimes g}^{\prime}(s, \chi)$ mentioned before this proof. We also use Deligne's estimate [3] repeatedly. We get

$$
F(s) \geq \frac{1}{2}-\frac{c\left(\left|\lambda_{2}\right|+\left|\lambda_{1}\right|\right)}{(\sigma-1)^{2}}\left(d_{1} d_{2}\right)^{c(1-\sigma)+\varepsilon}
$$

near $s=1$. We prove $L_{f \otimes g}\left((k+l) / 2, \chi_{2}\right)>0$ by applying the method of Ogg [11] (cf. the proof of Theorem 4 of [11]) to $F(s)$ in this case. Then we get $\lambda_{i} \ll L_{f \otimes g}\left((k+l) / 2, \chi_{2}\right)\left(d_{1} d_{2}\right)^{\varepsilon}, i=1,2$, for any $\varepsilon>0$. Here, for the estimate of $\lambda_{1}$, it is essential that the function $F(s)$ includes the square of $L_{f \otimes g}\left(s+(k+l) / 2-1, \chi_{2}\right)$ as a factor. Hence we obtain

$$
F(s) \geq \frac{1}{2}-\frac{c L_{f \otimes g}\left((k+l) / 2, \chi_{2}\right)}{(\sigma-1)^{2}}\left(d_{1} d_{2}\right)^{c(1-\sigma)+\varepsilon} .
$$

The proof in the case of $f \neq g$ is now finished.

The proof in the case of $f=g$ is easier, by applying the same method to

$$
\begin{aligned}
F(s)= & L_{f \otimes f}(s+k-1) L_{f \otimes f}\left(s+k-1, \chi_{1}\right) \\
& \times L_{f \otimes f}\left(s+k-1, \chi_{2}\right) L_{f \otimes f}\left(s+k-1, \chi_{1} \chi_{2}\right) .
\end{aligned}
$$

This function was already introduced by Golubeva-Fomenko [5].

3. The proof of Theorem 3. The method of proof is similar to the argument of Davenport [2]. We use the results of Section 2. We investigate 
the behaviour of

$$
\psi_{f, g}(x ; d, a)=\sum_{\substack{n \leq x \\ n \equiv a(\bmod d)}} \Lambda(n, f \otimes g), \quad(a, d)=1 \quad(x \geq 2),
$$

where

$$
\Lambda(y, f \otimes g)= \begin{cases}\left(\alpha_{p}^{m}+\bar{\alpha}_{p}^{m}\right)\left(\beta_{p}^{m}+\bar{\beta}_{p}^{m}\right) \log p, & y=p^{m}, m \in \mathbb{N}, \\ 0, & y \in \mathbb{R}, y \neq p^{m}, m \in \mathbb{N} .\end{cases}
$$

We consider

$$
\psi_{f \otimes g}(x, \chi)=\sum_{n \leq x} \Lambda(n, f \otimes g) \chi(n),
$$

where $\chi$ is a Dirichlet character modulo $d$. Then we get

$$
\psi_{f, g}(x ; d, a)=\frac{1}{\varphi(d)} \sum_{\chi} \bar{\chi}(a) \psi_{f \otimes g}(x, \chi),
$$

where $\varphi$ is the Euler function and the summation runs over all $\chi$ modulo $d$. Hence, we have to investigate the behaviour of $\psi_{f \otimes g}(x, \chi)$. From the Euler product of $L_{f \otimes g}(s, \chi)$ we get

$$
-\frac{L_{f \otimes g}^{\prime}}{L_{f \otimes g}}(s, \chi)=\sum_{n=1}^{\infty} \Lambda(n, f \otimes g) \chi(n) n^{-s} \quad(\sigma>(k+l) / 2) .
$$

Hence, defining $\chi(x)=0$ when $x \notin \mathbb{N}$, we find

$$
\psi_{0, f \otimes g}(x, \chi)=\psi_{f \otimes g}(x, \chi)-\frac{1}{2} \Lambda(x, f \otimes g) \chi(x),
$$

where

$$
\psi_{0, f \otimes g}(x, \chi)=\frac{1}{2 \pi i} \int_{h-i \infty}^{h+i \infty}\left(-\frac{L_{f \otimes g}^{\prime}}{L_{f \otimes g}}(s, \chi)\right) \frac{x^{s}}{s} d s \quad(h>(k+l) / 2) .
$$

Temporarily we assume that $\chi$ is a primitive character. Let $T>0$ and $h=(k+l) / 2+1 / \log x$. We argue analogously to Sections 17 and 19 of Davenport [2], using (2.3), the Lemma in the proof of Theorem 2 in Perelli $[12]$ and Theorem 1 . We get

$$
\begin{aligned}
\psi_{f \otimes g}(x, \chi)= & -\sum_{|\gamma|<T}^{\prime} \frac{x^{\varrho}}{\varrho}-\frac{x^{\beta_{1}}}{\beta_{1}} \\
& +O\left(x^{(k+l) / 2-3 / 4} \log x+\frac{x^{(k+l) / 2}}{T}(\log x d)^{2}\right),
\end{aligned}
$$

where $e \leq T \leq x, \beta_{1}$ is Siegel's zero of $L_{f \otimes g}(s, \chi)$ and the dash means that we exclude two non-trivial zeros $\beta_{1}$ and $k+l-1-\beta_{1}$ from the summation. 
We now consider the case where $\chi$ is a non-primitive character. If the primitive character $\chi^{*}$ induces $\chi$, then

$$
\left|\psi_{f \otimes g}\left(x, \chi^{*}\right)-\psi_{f \otimes g}(x, \chi)\right|=\left|\sum_{\substack{n \leq x \\(n, d)>1}} \Lambda(n, f \otimes g) \chi^{*}(n)\right| \ll \frac{x^{(k+l) / 2}}{T}(\log x d)^{2}
$$

by using Deligne's estimate [3]. Hence, the formula (3.1) is still valid.

Applying the same argument to $\psi_{f \otimes g}(x)=\sum_{n \leq x} \Lambda(n, f \otimes g)$, we get

$$
\psi_{f \otimes g}(x)=O\left(x^{(k+l) / 2} T^{-1}(\log x)^{2}+x^{(k+l) / 2} \exp (-c \log x / \log T)(\log T)^{2}\right) .
$$

This estimate yields the estimate of $\psi_{f \otimes g}\left(x, \chi_{0}\right)$, where $\chi_{0}$ is the principal character. Hence, under the condition $d \leq(\log x)^{M}$, we obtain

$$
\psi_{f, g}(x ; d, a)=O\left(x^{(k+l) / 2} \exp (-c \sqrt{\log x})\right)
$$

by choosing $T=\exp (c \sqrt{\log x})$ and using Theorems 1 and 2 . Theorem 3 now follows immediately.

\section{References}

[1] E. Carletti, G. Monti Bragadin and A. Perelli, On general L-functions, Acta Arith. 66 (1994), 147-179.

[2] H. Davenport, Multiplicative Number Theory, 2nd ed., Springer, 1980.

[3] P. Deligne, La conjecture de Weil I, Inst. Hautes Études Sci. Publ. Math. 43 (1974), 273-307.

[4] D. N. Goldfeld, A simple proof of Siegel's theorem, Proc. Nat. Acad. Sci. U.S.A. 71 (1974), 1055.

[5] E. P. Golubeva and O. M. Fomenko, Values of Dirichlet series associated with modular forms at the points $s=1 / 2,1$, J. Soviet Math. 36 (1987), 79-93.

[6] J. L. Hafner, On the representation of the summatory functions of a class of arithmetical functions, in: Lecture Notes in Math. 899, Springer, 1981, 148-165.

[7] J. Hoffstein and P. Lockhart, Coefficients of Maass forms and the Siegel zero, Ann. of Math. 140 (1994), 161-181.

[8] J. Hoffstein and D. Ramakrishnan, Siegel zeros and cusp forms, Internat. Math. Res. Notices (1995), 279-308.

[9] W. Li, L-series of Rankin type and their functional equations, Math. Ann. 244 (1979), 135-166.

[10] Ju. I. Manin and A. A. Pančiškin, Convolutions of Hecke series and their values at lattice points, Math. USSR-Sb. 33 (1977), 539-571.

[11] A. P. Ogg, On a convolution of L-series, Invent. Math. 7 (1969), 297-312.

[12] A. Perelli, General L-functions, Ann. Mat. Pura Appl. 130 (1982), 287-306.

[13] - On the prime number theorem for the coefficients of certain modular forms, in: Banach Center Publ. 17, PWN-Polish Sci. Publ., Warszawa, 1985, 405-410.

[14] A. Perelli and G. Puglisi, Real zeros of general L-functions, Rend. Accad. Naz. Lincei (8) 70 (1982), 67-74. 
[15] C. L. Siegel, Advanced Analytic Number Theory, Tata Inst. Fund. Res., Bombay, 1980.

Graduate School of Mathematics

Nagoya University

Chikusa-ku, Nagoya, 464-8602

Japan

E-mail: m96046i@math.nagoya-u.ac.jp

Received on 17.8.1998

and in revised form on 3.7.1999 